\title{
Chapter 1 \\ Overview and Evidence-Based \\ Recommendations to Address Health and Human Rights Inequities Faced by Sex Workers
}

\author{
Shira M. Goldenberg, Ruth Morgan Thomas, Anna Forbes, and Stefan Baral
}

Based on a powerful combination of sex worker community and academic evidence, this edited volume highlights the unacceptable health and social inequities that sex workers in all their diversity continue to face across diverse global and policy contexts. The work presented here was guided by a unique community-academic partnership, developed to ensure that sex workers' voices were amplified in describing challenges, and presenting solutions and ways forward for research, service delivery, and policymaking. Interdisciplinary author teams comprised of academic researchers, sex workers, and sex worker-led organisations collaboratively developed each chapter to synthesise research evidence as well as highlight lessons learned from local-level experiences across different regions.

This edited volume aims to provide a comprehensive overview of health and human rights inequities faced by sex workers around the world. It aims to articulate structural determinants of sex workers' health and occupational outcomes (e.g. legislation, migration, healthcare delivery settings) and describe evidence-based interventions and 'best practices', including the roles of full decriminalisation of sex work, community empowerment models, and multi-level and integrated intervention

\footnotetext{
S. M. Goldenberg $(\bowtie)$

Faculty of Health Sciences, Simon Fraser University, Burnaby, BC, Canada

Centre for Gender and Sexual Health Equity, Vancouver, BC, Canada

e-mail: Shira.Goldenberg@cgshe.ubc.ca

R. Morgan Thomas

The Matrix, Global Network of Sex Work Projects - NSWP, Edinburgh, UK

e-mail: ruth.morganthomas@nswp.org
}

\author{
A. Forbes \\ Independent Consultant, Kensington, MD, USA \\ S. Baral \\ Center for Public Health and Human Rights, Department of Epidemiology, \\ Johns Hopkins University, Baltimore, MD, USA \\ e-mail: sbaral@jhu.edu
}


approaches. The chapters describe an elevated burden of HIV and sexually transmitted infections and drug-related harms; persistent and unacceptable experiences of violence and other human rights violations; and significant unmet sexual and reproductive health needs. Importantly, this edited volume also demonstrates that sex workers are not passive recipients of such structural inequity and violence, but rather actively resist and demonstrate tremendous resilience in the face of these harms. Sex workers across diverse global settings continue to mobilise to advocate for improved health, safety, and human rights conditions and policy changes - such 'community empowerment' interventions have been identified as a best practice for improving sex workers' health and labour rights.

\section{Part I: Burden of Health and Human Rights Inequities Faced by Sex Workers Globally}

In Chap. 2, authors Viswasam, Rivera, Comins, Rao, Lyons, and Baral draw on epidemiological studies and the lived experience of sex workers to describe the global HIV burden among sex workers and the factors that influence this. It also describes coverage of and gaps in interventions to reduce HIV-related inequities faced by sex workers over the past decade. This chapter shows that globally, sex workers face a disproportionate burden of HIV, and continue to face unacceptable gaps in access to HIV prevention, treatment, care, and support services. It also highlights current gaps in data, including limited research with communities of cis-men and transgender sex workers, as well as the need for further data focused on the harms of criminalisation and intersectional risks. Key recommendations include national action towards full decriminalisation of sex work and scale-up of community empowerment approaches, which remain vital alongside and in tandem with emerging biomedical HIV prevention approaches.

Although violence and other human rights violations are recognised as critical public health and human rights violations, global literature on violence against sex workers remains scant. In Chap. 3, authors Argento, Win, McBride, and Shannon illustrate this through a global overview of literature on violence and other human rights violations faced by sex workers, complemented by case studies from community partners in the Asia Pacific region. This chapter highlights the need for legislative reforms to decriminalise all aspects of sex work, end impunity for those who commit violence against sex workers, and ensure that sex workers have legally enforceable rights to occupational health and safety protections. The authors indicate the need for political commitment to reduce broader structural inequities, uphold anti-discrimination and other rights-respecting laws, and reduce stigma and exclusion. They also call for increased funding to support scale-up of community-led empowerment approaches and rights-based strategies to mitigate risk of violence, enable safer work environments, and uphold human rights among sex workers globally.

Chapter 4 addresses sex workers' lack of access to sexual and reproductive health and rights (SRHR). In this chapter, authors Shapiro and Duff draw on academic 
evidence and community consultations undertaken by NSWP with sex workers across ten countries to examine barriers to achieving SRHR for sex workers. Findings indicate that whereas HIV and STIs have been a primary focus of many sex worker services and interventions, sex workers face a high burden of unmet sexual and reproductive health services across diverse contexts. Multiple factorsincluding criminalisation of sex work, a lack of sex worker-specific SRHR models, and the stigmatisation of same-sex relationships and gender non-conformancecontribute to barriers to achieving SRHR. The authors recommend: increasing funding and support for comprehensive SRHR services designed to meet the needs of sex workers of all genders; ensuring access to safe, legal, affordable, and noncoercive SRHR services, including abortion; integrating SRHR with HIV and STI services as a 'one-stop-shop' model; promoting community-led SRH education programming for sex workers and their clients; and prioritising broader efforts towards full decriminalisation of sex work, community empowerment models of SRH care, and anti-stigma and discrimination efforts.

Work by Logie, Wang, Lalor, Levermore, and Williams in Chap. 5 addresses sex workers' mental health, examining mental health outcomes in relation to social cohesion among sex workers. In collaboration with two sex worker-led organisations in Jamaica, the findings generated from research with 340 women, men, and gender-diverse sex workers found that enhanced social cohesion was linked to reduced depressive symptoms and violence among participants, thus promoting their health and safety. Based on these findings and review of the literature, the authors describe an urgent need for full decriminalisation of sex work and samegender sexual practices to advance health and human rights, alongside increased screening for mental health and scale-up of access to mental health services for mitigating the effects of structural stigmas.

In Chap. 6, authors Iversen, Long, Lutnick, and Maher evaluated the health needs of sex workers who use drugs, using a systematic review and community case studies from the St. James Infirmary in San Francisco. This systematic review of 86 studies conducted in 46 countries reveals a pooled prevalence of lifetime illicit drug use among sex workers of $35 \%$, with $29 \%$ lifetime pooled prevalence among female sex workers. The authors note significant gaps existing in the data quality and availability in this review. Key recommendations included an urgent need for future research in partnership with sex workers to improve the quality and quantity of data on illicit drug use among sex workers and guide the creation, implementation, and evaluation of programmes and services that meaningfully address the needs of this population.

\section{Part II: Structural Determinants of Health and Human Rights Inequities in Sex Work}

Work by Krüsi, D'Adamo, and Sernick in Chap. 7 illustrates how the criminalisation and policing of sex work shapes sex workers' health and safety in the context of different legislative frameworks governing sex work around the world. Based on 
synthesis of research evidence and sex work community case studies from the global North and South, this chapter shows how sex workers' occupational health, safety, and human rights are violated and undermined under various criminalised models. These models include full criminalisation, as well as 'end-demand' models that criminalise clients and third parties but not sex workers directly. This chapter shows how numerous legislative frameworks sanction and perpetuate structural violence and negative health outcomes among sex workers. This chapter concludes with an evidence-based call for the full decriminalisation of all aspects of sex work, and also highlights the need to include sex workers from all segments of the sex industry, including those who are marginalised due to racialisation, im/migration status, and illicit substance use, in evidence-based policymaking.

In Chap. 8, authors Richter and Buthelezi describe experiences of stigma, denial of care, and other human rights violations faced by sex workers within health service delivery settings in Africa. They powerfully show that sex workers' negative experiences with health services act as a severe barrier to the right to health and quality health care-by inhibiting effective treatment, prevention and support for HIV and other health-related needs of sex workers, including sexual and reproductive health, preventative care, and mental health. In particular, stigmatising and discriminatory treatment by healthcare workers and non-clinical staff have a farreaching negative impact on sex workers, undermining their wellbeing and access to care. In contrast, positive interactions with healthcare providers and health services empower sex workers, affirm sex worker dignity and agency, and assist in cultivating healthy behaviour and improved health outcomes. The authors conclude with recommendations for comprehensive, rights-affirming health programmes designed in partnership with sex workers, as well as programmes that focus on strategic and practical sex worker needs in the African context; these programmes should include structural interventions to shift away from outdated criminalised legal frameworks and implement violence prevention strategies, psycho-social support services, sex worker empowerment initiatives, and sex workerled programmes.

Chapter 9 focuses on the health and social needs of women, men, and genderdiverse im/migrant sex workers. Authors McBride and Janushev draw on research evidence and community consultations in Europe to showcase the unique concerns of im/migrant workers in destination settings, based on their intersecting identities as sex workers as well as im/migrants. These concerns include racialised police harassment and surveillance, mandatory health testing, economic marginalisation, discrimination, and language barriers. Results of community consultations, primarily among male and gender-diverse im/migrant sex workers, illustrate a range of obstacles created by such social and structural exclusion, as well as the resilience of $\mathrm{im} /$ migrant workers in their efforts to resist them. Recommendations for policies and programmes include supporting safer indoor sex work environments, removal of punitive sex work, immigration, and public health laws and policies that affect $\mathrm{im} /$ migrant sex workers, and supporting non-stigmatising, sex worker-led education, outreach, and services. 


\section{Part III: Evidence-Based Services and Best Practices: Opportunities for Action}

In Chap. 10, work by Abel and Healy draw on academic research and experiences of the New Zealand Prostitutes Collective to compellingly illustrate how New Zealand's national decriminalisation of sex work promotes best practices for occupational health, safety, and the broader social inclusion of sex workers. In stark contrast to criminalised settings, where sex workers fear coming forward to report violence due to mistrust of police and fear that they or their workplaces may be prosecuted, this work reports improved access to justice and police responsiveness to violence against sex workers under decriminalisation. Furthermore, this chapter reveals that decriminalisation promotes interagency collaboration to support safer indoor work environments and helps to ensure that new sex workers are well informed about safe and legal sex work practices. Nonetheless, gaps are noted, in particular for migrant workers who are not protected under this law. The authors conclude with recommending full decriminalisation of sex work that is also inclusive of im/migrant workers, so that all sex workers may benefit from improved occupational health, safety, and agency.

Chapter 11 describes the ways in which community empowerment and mobilisation strategies have, in the last few decades, become critical tools used by sex workers in many settings to confront the health and human rights challenges they face, including HIV, violence, discrimination, and labour rights abuses. In this chapter, authors Navarrete Gil, Ramaiah, Mantsios, Barrington, and Kerrigan review the literature on community empowerment and mobilisation, highlighting their effectiveness with case studies developed by sex work community organisations APROASE in Mexico and Ashodaya Samithi in India. The authors also identify several key evidence gaps, including a lack of systematic community empowermentbased approaches and evaluation of these efforts for male and transgender sex workers. Key recommendations include efforts to take community empowerment efforts to scale and to conduct additional longitudinal evaluation research assessing longterm changes across diverse contexts.

Features of sex workers' occupational environments are known to greatly shape health outcomes. In Chap. 12, authors West, Hilton, Montgomery, and Ebben describe the health and safety challenges, protective factors, and unique considerations and opportunities associated with indoor sex work. A case study from Empower in Thailand, a sex worker-led organisation, outlines the physical and policy components essential to ensuring sex workers' rights, occupational safety, and health in an indoor workplace. The authors conclude indoor venues pose important potential for establishing and implementing occupational health and safety standards in sex work, and also may provide substantial opportunity for collective organising given the close proximity of people working together. However, any efforts to improve the health and safety of sex workers must explicitly address the structural conditions that lead to power imbalances and which undermine sex worker agency and equality. Key recommendations include promoting occupational 
health and safety within indoor spaces, prioritising sex worker leadership and inclusion through community mobilisation and engagement efforts, and advocacy and policy reform to remove punitive policies.

Finally, in Chap. 13, Schwartz, Viswasam, and Abdalla highlight that the intersecting and multi-factorial factors that influence sex workers' health in most settings are too complex to be addressed with single disease-focused interventions. This chapter synthesises scientific evidence on existing approaches to designing and evaluating multi-level and integrated interventions to improve sex workers' health, which is complemented by sex work community perspectives from the Kenya Sex Workers Alliance (KESWA). This chapter highlights the necessity for integrated, multi-level public health approaches-including tailored combinations of structural, behavioural, and bio-medical interventions - to promote improved health outcomes for sex workers (e.g. HIV, gender-based violence, SRHR). The chapter also discusses challenges and considerations in the development and evaluation of such interventions, including the need for additional support and prioritisation for meaningful sex worker involvement. The chapter concludes with recommendations for further intervention research that incorporates and evaluates structural intervention components, as well as the critical need to support increased opportunities for leadership from the sex worker community in setting and implementing this research agenda.

\section{Implications for Policy, Advocacy, and Programmes}

Ten high-level, evidence-based recommendations for policy, programmes, and research informed by the chapters in this edited volume are presented in Box 1.1. We urge policymakers, service delivery organisations, researchers, community organisations, and other implementing partners to implement these recommendations in policy, programmes, and research efforts in the area of sex workers' health and human rights.

\section{Box 1.1 Key Evidence-Based Recommendations of this Edited Volume}

1. Decriminalise all aspects of sex work.

Governments, policymakers, and advocates must actively pursue the full decriminalisation of sex work, including sex workers, clients and third parties, and the removal of all punitive laws and policies that increase the vulnerabilities of different sex worker sub-populations. Criminalisation is a primary driver of the stigma and discrimination experienced by sex workers when accessing health services and a major reason for why they continue to be disproportionately affected by HIV. Criminalisation also deters sex workers from reporting crimes to the police and exacerbates abuse and violence from law enforcement officers, as well as enabling 
Box 1.1 (continued)

impunity of all perpetrators of abuse and violence. Criminalisation of sex work, HIV transmission, and same-sex sexual activity increases stigma and discrimination and causes fear among sex workers that prevents them from seeking the health care they need. Decriminalisation of all aspects of sex work would significantly reduce the barriers to health for sex workers, in terms of both access to health care and meaningful involvement in the development of health services aimed at them. It would also enable occupational health and safety standards to be rolled out in ways that promote the safety, agency, and health of sex workers.

2. Recognise sex work as work.

Governments should extend all legal protections and labour rights, to which all workers are entitled, to sex workers and remove legal and policy barriers that deny sex workers the protection of employment legislation, including health and safety protections and anti-discrimination, and access to social protection schemes.

3. Ensure that healthcare services are accessible, non-coercive, and responsive to the diversity of sex workers' needs.

Health is not only the absence of illness, it is wellbeing, and sex workers in all their diversity need accessible, non-stigmatising access to health care that comprehensively fulfils their right to health. This includes access to non-coercive and non-discriminatory sexual and reproductive health services, psychological and psycho-social support, specialist health services, as well as access to justice and social protection. Sex workers are not vectors of disease and must not be treated as such by the health system; rather, they are on the frontlines of prevention and are contributing members of society. Integrated models of service delivery are often more convenient and less stigmatising than individual diseaseoriented models that focus more narrowly on specific conditions (e.g. HIV).

4. End police, immigration, and other state-sanctioned crackdowns, surveillance, and harassment of sex workers, third parties, and clients.

Alongside longer term efforts towards full decriminalisation, policies that address the harms of laws, policies, and practices surrounding sex work and immigration are urgently needed. There is a need for the implementation of local or regional-level 'non-harassment' and 'access without fear' policies that end police and immigration crackdowns, public health surveillance, arrests and prosecutions targeting im/migrant and non-migrant sex workers, third parties, and clients. This allows efforts to shift away from punitive measures that have been shown to severely undermine sex workers' health and violate their rights, towards community-led efforts that will reach and support those most in need. 
Box 1.1 (continued)

5. Build trust and partnerships between the health system and sex worker-led organisations.

The organisation and delivery of healthcare services should treat sex workers as experts on their own lives and their health needs, and as potential service providers, not just service users. Non-stigmatising, nondiscriminatory approaches are best developed by, for, and with sex workers and sex worker-led community organisations. To build more nuanced and non-stigmatising relationships between healthcare providers and sex workers, ongoing community-led training for providers regarding sex workers' lived experiences, expertise, and needs is recommended. Understanding health inequities and the needs of key populations, including sex workers, should also be core components of medical education.

6. Prioritise community empowerment and mobilisation in research, programming, and policy.

Sex workers and sex worker-led organisations are the experts on their own lives and are ideally situated to inform approaches and deliver services that support their needs. Community-based and sex worker-led models of health and social services delivery are recognised 'best practices'for meeting sex workers' needs but are often not prioritised by funding agencies and health systems. Community empowerment and mobilisation should be implemented as critical strategies for advancing rights-affirming and evidence-based interventions including full decriminalisation, non-coercive occupational health and safety standards, addressing violence, ensuring access to justice and broader anti-stigma efforts.

7. Support partnership and collaboration between sex worker-led organisations and academics, policymakers and programmers committed to addressing inequities across health, human rights, and justice.

These efforts are vital to ensuring balanced and inclusive perspectives across research, policymaking, and practice that meaningfully advance sex workers' health and human rights. It is often particularly relevant to collaborate with community-led organisations that work for other marginalised and criminalised groups that are disproportionally represented within sex work, such as im/migrants, people living with HIV, people who use drugs, lesbian, gay and bisexual, and transgender people, Black, Indigenous, and other People of Colour (BIPOC), and women that share the core values of the sex worker rights movement.

8. Ensure access to health and social services and supports for sex workers, regardless of internal mobility or immigration status.

Sex workers require access to critical social services, including eligibility for housing, food support, basic income and employment insurance support, and other vital social services, including support for survivors of 
Box 1.1 (continued)

violence. Despite this need, im/migrant and mobile sex workers are often unjustly excluded from these due to restrictions on eligibility, stigma, and the lack of recognition of sex work as work. Urgent policy reforms are especially needed to ensure these protections for im/migrant and mobile workers, who are often unable to access health and social services and protection schemes that are dependent on local registration and/or immigration status.

9. Support sex worker-led, resilient, and evidence-based approaches. Sex workers show individual and collective resilience, adaptability, and resistance in their work, lives, and advocacy efforts. Recognition of these strengths and efforts to build on sex worker communities knowledge and expertise in programmes, policy, and research are recommended. It is also important to ensure that research focused on health and social inequities faced by sex workers consider sex workers as agents and experts in their own lives.

10. Ensure resources are made available to strengthen capacity among sex worker-led organisations to become meaningfully involved in research, policy, and programmes.

These efforts are vital to meaningful and successful community empowerment and mobilisation efforts, yet they remain woefully under-resourced. Many sex worker-led organisations are already engaging in these efforts and simply lack the funding to bolster them.

As we finalise this edited volume, we are living in a pandemic that has further exposed and exacerbated the inequities and vulnerabilities that confront sex workers of all genders around the world. Sex work is a work, and sex workers around the world have faced total or near-total loss of income in the context of the COVID-19 pandemic. Despite sex workers being recognised as informal workers by the international Labour Organisation, the criminalisation of sex work has resulted in many governments taking disproportionate repressive measures against sex workers in response to COVID-19, including increasing raids and arrests, compulsory testing, threats of deportation, and bulldozing their homes. In the United States, despite sex work being legal in some states, sex workers are explicitly excluded from social protection offered to other workers. It is imperative that states recognise sex workers, in all their diversity, and ensure that emergency social protection schemes are accessible to them.

Health inequities have long defined health and the healthcare access for sex workers around the world. The chapters show here that while there have been significant investments in clinical and epidemiologic research on sex workers, sex workers' health, and human rights continue to be hampered by limited attention and investment in structural interventions. As seen here, the average health and human 
rights indicators of sex workers remain suboptimal in 2020, a finding at least in part explained by inequity in access to health care and justice. In this context, COVID-19 has rapidly emerged as a major global threat to health. While it was initially thought that the pandemic would be the great equaliser as it would not discriminate, it is clear that COVID-19 incidence and mortality have rapidly reinforced health inequities drawn by historical and contemporary injustices. Throughout this book, we synthesised the data highlighting specific health and social inequities among sex workers driven by criminalisation and other structural factors. In the absence of major policy changes, such inequities will likely only be reinforced during COVID-19.

Ultimately, advancing the COVID-19 response and saving lives requires that we rapidly address inequities affecting sex workers rather than ignoring, or worse, reinforcing them. The COVID-19 pandemic provides a stark example of how sex workers are too often excluded from emergency responses and social protection schemes. COVID-19 has also clearly shown the critical contributions of sex worker-led organisations, who wherever and whenever possible, have launched and implemented emergency responses and mutual aid funds for their communities.

In April 2020, a joint statement was issued [1] by the Global Network of Sex Work Projects (NSWP) and the Joint United Nations Programme on HIV/AIDS (UNAIDS), which noted the severe inequities and hardships faced by sex workers during the current COVID-19 pandemic and called on countries to take the following comprehensive set of actions to mitigate these:

- Access to national social protection schemes for sex workers, including income support schemes.

- An immediate firewall between health services and immigration authorities in order to ensure that migrant sex workers can access health services.

- Emergency financial support for sex workers facing destitution, particularly migrants who are unable to access residency-based financial support.

- An immediate end to evictions and access to appropriate emergency housing for homeless sex workers.

- Stopping raids on sex workers' homes and sex work premises and ensuring that all measures to protect public health are proportionate.

- An immediate halt to arrests and prosecutions for sex work-related activity, moving away from punitive measures and criminalisation towards reaching and serving those most in need.

- An immediate end to the use of criminal law to enforce COVID-19-related restrictions, including forced COVID-19 testing and related prosecutions.

- Automatic extensions on visas due to expire as travel restrictions tighten. Immigration detention systems must support detainees in safe accommodation.

- The engagement of sex worker communities in responses-the meaningful involvement of sex worker-led organisations in emergency public health planning groups. 
If we are to address the ongoing inequities highlighted throughout all the chapters in this edited volume, then sex worker's human rights must be protected, respected, and fulfilled. Fundamental changes are needed. Policymakers must be willing to listen to sex workers in all their diversity, and to review existing policy and legislation to ensure the harms identified in each chapter are eradicated. Sex workers' voices must be amplified and respected in decision-making that directly affects their lives and work. Policies and programmes must ensure that the agency and bodily autonomy, as well as the livelihoods of sex workers, are not undermined. It is essential that sex workers are meaningfully involved from the very start of processes to develop or review policies and programmes, to ensure existing harms are considered and mitigated. Change will also require the recognition of the critical role that sex worker-led organisations should play in addressing the inequities and identifying sustainable rights-affirming responses that uphold the value and dignity of sex workers, which must be appropriately resourced.

\section{Reference}

1. UNAIDS and NSWP. Sex workers must not be left behind in the response to COVID-19 [press release]; 8 Apr 2020.

Open Access This chapter is licensed under the terms of the Creative Commons Attribution 4.0 International License (http://creativecommons.org/licenses/by/4.0/), which permits use, sharing, adaptation, distribution and reproduction in any medium or format, as long as you give appropriate credit to the original author(s) and the source, provide a link to the Creative Commons license and indicate if changes were made.

The images or other third party material in this chapter are included in the chapter's Creative Commons license, unless indicated otherwise in a credit line to the material. If material is not included in the chapter's Creative Commons license and your intended use is not permitted by statutory regulation or exceeds the permitted use, you will need to obtain permission directly from the copyright holder.

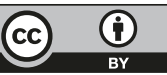

